\title{
ORIGINAL RESEARCH \\ Stent-Supported Aneurysm Coiling: A Literature Survey of Treatment and Follow-Up
}

M. Shapiro

T. Becske

D. Sahlein

J. Babb

P.K. Nelson

BACKGROUND AND PURPOSE: Stent-supported aneurysm coiling has been utilized with increasing frequency over the past few years, particularly for addressing treatment of complex and wide-neck aneurysms. A sizable body of literature describing various experiences with stent-supported coiling now exists. The purpose of this research was to carry out a comprehensive literature survey of stent-supported aneurysm coiling.

MATERIALS AND METHODS: A survey of papers reporting results with stent-assisted aneurysm coiling through January 2011 was conducted to identify the prevalence of stent-related and other complications, occlusion rates, and clinical and angiographic follow-up data.

RESULTS: Thirty-nine articles with 1517 patients met inclusion criteria for initial analysis, follow-up analysis, or both. Overall, $9 \%$ of cases were confounded by a technical stent-related issue, including $4 \%$ failure of deployment. The overall procedure complication rate was $19 \%$, with periprocedural mortality of $2.1 \%$. Approximately $45 \%$ of aneurysms were completely occluded at first treatment session, increasing to $61 \%$ on follow-up. Approximately $3.5 \%$ in-stent stenosis and $0.6 \%$ stent occlusion were observed at angiographic follow-up. Delayed stroke or transient ischemic attack was reported in $3 \%$ of subjects. Within a subset of articles, the incidence of stent-related issues in the first 10 patients was significantly higher than in subsequent subjects, supporting the notion of a procedural "learning curve."

CoNCLUSIONS: In experienced hands, the morbidity of stent-supported coiling is somewhat higher compared with "traditional" coiling. As might be expected, execution of the procedure appears improved with experience. Complete occlusion rates remain somewhat low. More and longer term angiographic follow-up information is needed to understand delayed stent-related issues and to better define the durability of treatment.

ABBREVIATIONS: $\mathrm{OR}=$ odds ratio

E ndovascular treatment of cerebral aneurysms with detachable coils has become increasingly adopted over the past 2 decades. The widespread acceptance of endosaccular coiling however has been dampened by the likelihood of aneurysm recurrence concerns over the durability of treatment and the significance of incompletely treated aneurysms. ${ }^{1}$ These issues are, ironically, most relevant to the subgroup of complex and large aneurysms for which coiling was initially used. Yet another group of fusiform and wide-neck aneurysms remained unaddressed by reconstructive surgical and endovascular techniques until the introduction of dedicated intracranial stentlike devices, such as Neuroform (Boston Scientific, Natick, Massachusetts), Enterprise (Cordis, Miami Lakes, Florida), Solitaire (ev3, Irvine, California), and LEO (Balt Extrusion, Montmorency, France). Stent deployment across the aneurysm neck, followed immediately or in delayed manner, by coil packing of the aneurysm, has slowly been more widely adopted, particularly for complex aneurysms, with the intention to promote improved coil packing densities and greater stability of the coil mass within the aneurysmal sac; hypothet-

Received February 16, 2011; accepted after revision April 25.

From the Bernard and Irene Schwartz Neurointerventional Radiology Center, Departments of Radiology (M.S., T.B., D.S., J.B., P.K.N.), Neurology (M.S., T.B., D.S.), and Neurosurgery (P.K.N.), NYU Langone Medical Center, New York, New York.

Please address correspondence to Maksim Shapiro, NYU Medical Center, Department of Radiology, Tisch Hospital, 560 First Ave, HE 208, New York, NY 10016; e-mail: shapim06@med.nyu.edu

三E Indicates article with supplemental on-line table.

http://dx.doi.org/10.3174/ajnr.A2719 ically reducing the likelihood of neck recurrence and coil compaction through some hemodynamic modification of aneurysmal blood flow and facilitation of intimal overgrowth of the implant and neck region coils. Although the availability of these devices has enabled the endovascular treatment of previously uncoilable and inoperable aneurysms, the use of stentassisted coiling has brought with it other important considerations, including the necessity of aggressive antiplatelet therapy that limits its application in the setting of subarachnoid hemorrhage, and delayed stent-related issues such as the development of in-stent stenosis and parent vessel occlusion.

The purpose of the present study was to review the literature on stent-assisted coiling to ascertain the frequency of stent-related complications and to assess the impact of the technique on the angiographic and anatomic outcome of endovascular aneurysm treatment.

\section{Materials and Methods}

Medline and PubMed searches were performed through January 2011 to identify relevant articles by using search strings of Neuroform, Enterprise, LEO, intracranial stent, aneurysm coiling rupture, aneurysm coiling thrombosis, cerebral stent, and intracranial aneurysm stent from 1999 to 2011. A Google search by using the string "intracranial aneurysm stent" also yielded several not otherwise identified articles. Additional sources were culled through bibliographies of existing articles. Information derived from discovered articles was used for overall procedure complication analysis and, when available, for specific long-term follow-up metrics. Articles selected for overall procedure analysis were required to contain information on the total 
number of subjects in the study and overall number of complications and procedure-related deaths. Additional information, available in most publications, was collected regarding the following: 1) type of complication encountered (eg, thromboembolic, hemorrhagic); 2) fraction of patients in whom the stent could not be delivered (considered as a stent-related technical complication) or where any issue was encountered with a deployed stent (eg, malposition, migration); and 3) aneurysm size, rupture status, location, and initial posttreatment occlusion.

Articles with fewer than 5 cases were excluded.

Most papers fulfilling the above-mentioned criteria also contained information on some form of patient follow-up. In addition, some publications not meeting the above-mentioned criteria for overall treatment analysis were used exclusively for follow-up calculations and included clinical (length, incidence of death, or TIA/ stroke in the relevant brain territory) and imaging data (aneurysm occlusion, recanalization, retreatment, in-stent stenosis/occlusion).

In some instances, publications from an individual center or centers contained potentially duplicate patient data. A single publication providing the most comprehensive dataset was then selected.

Ten publications ${ }^{2-11}$ contained data, usually in table format, on each patient within the study. In such cases, we collected information on total complications, stent complications, and death in the first 10 patients, and compared these parameters with those of subsequent patients in the same study for a "learning curve" analysis. For this component of the study, a meta-analysis was conducted to compare the first 10 subjects treated with subsequent patients in terms of the percentage of subjects manifesting overall complications, death, overall stent problems, stent delivery problems, or other stent problems. A separate analysis was conducted for each of these comparisons. Both random and fixed effects models were evaluated. Under the fixed effects model, it is assumed that all studies come from a common population and that the effect size, represented as an OR, is not different among the different studies. This assumption was tested by a heterogeneity test based on the Q statistic. If this test yields a significant result, then the fixed effects model may be invalid. In this case, the random effects model may be more appropriate, in which both the random variation within the studies and the variation between the different studies is incorporated. For the meta-analysis of each outcome measure, the comparison was based on the Mantel-Haenszel method for calculating the weighted summary OR under the fixed effects model. For each outcome measure, the heterogeneity statistic $\mathrm{Q}$ was then incorporated to calculate the summary OR under the random effects model. In the analysis for each outcome measure, only studies providing relevant data for both groups were included in the meta-analysis to avoid confounding of differences between studies with differences between groups. Statistical significance was defined as $P<.05$. MedCalc version 10.4.0.0 (Frank Schoonjans, Mariakerke, Belgium) was used for all computations.

No institutional review board clearance is required at our center for literature surveys.

\section{Results}

\section{Overall Data}

In total, 39 articles ${ }^{2-40}$ with 1517 patients met inclusion criteria for overall treatment, follow-up analyses, or both. Thirtyseven of these articles, reporting on 1510 aneurysms in 1457 patients, contained data for both overall procedure and specific follow-up data analysis. Two articles ${ }^{37,38}$ were used exclu- sively for follow-up analysis. The results are provided in Online Table 1. As evident from the table, only overall complication data were required to be present in every article. Additional parameters (eg, aneurysm size, initial occlusion) were not present in all publications. Calculations by using these parameters were carried out based on the number of subjects or cases within the relevant parameter dataset, and not on the overall subject or case number. Thus, the percentages listed in On-line Table 1 may not be deducible from overall figures provided in the table.

\section{Complications}

The overall complication incidence was $19 \%$, with an overall death incidence of $2.1 \%$. Thromboembolic issues were most prevalent at close to $10 \%$, leading to death in $0.6 \%$ of overall cases. Hemorrhagic complications occurred in $2.2 \%$ of cases but carried a higher association with mortality, accounting for $0.9 \%$ of overall deaths. Coil-related technical issues were infrequent $(2 \%)$ and almost always asymptomatic.

Stent-Related Technical Complications. Overall, 9\% of cases were associated with technical stent-related failure. Of these, the stent could not be deployed in $4 \%$. The remaining $5 \%$ included various issues related to an already deployed stent, such as migration and premature or misplaced deployment.

\section{Initial Occlusion}

Approximately $45 \%$ of aneurysms were deemed completely occluded at immediate postprocedure angiography. Due to heterogeneity in outcome reporting, we found no consistent method of categorizing near-complete, subtotal, incomplete, or residual occlusions into a quantifiable entity.

\section{Size}

Overall, approximately $63 \%$ of aneurysms measured $<10 \mathrm{~mm}$ in diameter. No stratification of initial or follow-up angiographic occlusion rate according to size was feasible.

\section{Rupture Status}

Twenty-two percent of aneurysms were treated in the setting of acute or relatively recent subarachnoid hemorrhage. No breakdown of complication incidence with respect to rupture status was possible.

\section{Clinical Follow-Up}

Twenty-seven papers ${ }^{2-5,7,9-11,13,15-18,20,24,25,28-30,33-40}$, containing 736 patients, reported clinical follow-up of 13-month average duration and 6-month median duration. Twenty-one patients $(3.2 \%)$ suffered delayed TIA/stroke in the territory subserved by the stented vessel.

\section{Imaging Follow-Up}

Twenty-three articles ${ }^{2-5,7,9,11,15-18,24,25,30,32-40}$ with 695 patients provided imaging (almost entirely catheter angiogrambased) follow-up data, reporting a $61 \%$ rate of complete aneurysm occlusion at variable follow-up times. Among these reports, $14 \%$ of aneurysms initially scored as completely occluded and subsequently recanalized, and $23 \%$ of aneurysms 
initially judged incompletely treated on the immediate posttreatment angiograms progressed to complete occlusion. Sixty-two patients (13\%) underwent retreatment. In-stent stenosis was observed in 24 cases $(3.5 \%)$ at an average 5 months of follow-up. Of these, 4 stenoses (17\%) were symptomatic. Delayed parent vessel/stent occlusion was noted in 4 cases $(0.6 \%$, 3 asymptomatic, 1 stroke), at 8 months average follow-up.

\section{Learning Curve Analysis}

From the original dataset, 10 articles $^{2-11}$ provided information that allowed determination of complications, outcome, and stent delivery data for each individual patient within the study, enabling comparison of clinical and technical complication rates between early and late experiences. The results are listed in Table.

\section{Discussion}

We found a large heterogeneous body of literature describing stent-assisted coil embolization of cerebral aneurysms, consisting of mostly retrospective cases series. The heterogeneity in adverse outcome reporting was particularly evident, precluding accurate subdivision of adverse event categories into clinically useful entities such as permanent deficit or transient deficit, whereas mortality data were more consistently present.

Ideally, we would have liked to perform a statistical comparison of complication rates in stent-assisted coiling to that of "traditional" coiling. We have previously carried out a similar analysis by comparing balloon-assisted and traditional coiling. ${ }^{41}$ However, such an analysis, to be statistically rigorous, requires that each publication contributing to the metaanalysis contain cases of both stent-assisted and traditional coiling treatment within the same study. To date, however, most publications reporting experience with stent-supported aneurysm treatment have no such "control" arm, precluding meaningful statistical analysis.

In a more qualitative sense, the overall complication rate of $19 \%$, as determined by our analysis of predominantly unruptured aneurysm treatment, is somewhat higher than that reported by most large single-center series. ${ }^{42,43}$ This seems to stem mainly from a higher incidence of thromboembolism. Despite the perceived increased bleeding risk associated with dual antiplatelet use in stented patients, the prevalence of hemorrhagic complications was more comparable with those reported for traditional coiling of predominantly unruptured aneurysms. ${ }^{41-43}$ A distinction among etiology of hemorrhage is also worthwhile in that traditional coiling is associated with predominantly subarachnoid hemorrhage due to periprocedural aneurysmal perforation, whereas stent-supported bleeding is a mixture of subarachnoid and parenchymal hemorrhage.

A relatively large percentage of patients (22\%) were treated in the setting of acute or recent subarachnoid hemorrhage, a factor well known to increase both overall, ${ }^{41,44}$ and particularly hemorrhagic ${ }^{44-46}$ treatment risk. Because no subset complication analysis with respect to aneurysm rupture status was feasible as a result of the tendency of most publications to report combined statistics, it is unclear to what extent these "ruptured" patients have contributed to overall complication incidence. However, as suggested by other studies of aneurysm coiling, ${ }^{47}$ the rupture disposition of an aneurysm has been shown to be highly correlated with treatment-related complications, and it may be reasonable to assume that a similar correlation exists for stent-assisted coiling.

Stent-related technical issues were an important focus of attention in our analysis. Again, we found that categorization of stent complications was limited both by range and prevalence of potential issues and by variability in their description. Eventually, we decided to subdivide stent-related technical issues into categories of 'delivery failure' and 'other', with 'other' containing any untoward technical occurrence among patients in whom a stent was deployed.

The learning curve analysis, we believe, clearly demonstrates the significance of operator experience in the overall success of treatment. To some unquantifiable extent however, this experience factor is probably diluted by concurrent evolution in stent technology.

The success of aneurysm occlusion, both initial and followup, is a particularly difficult parameter to analyze and interpret, and is subject to heterogeneity in grading practices and operator bias. Furthermore, its comparison to similar metrics of coiling series is suspect in that the target aneurysm populations are unlikely to be similar (with stent-assisted coiling typically being used for more complex aneurysms). The overall number of complete occlusions represents an aggregate of cases with varied aneurysm size and morphology. Therefore, we feel that direct comparison of occlusion rates between stent-assisted and traditional coiling is apt to be misleading unless various morphologic parameters can be controlled. Such an analysis is beyond the capability of a retrospective literature review. The overall numbers, particularly regarding incidence of retreatment and progressive thrombosis, can nevertheless be useful in terms of patient counseling and procedural planning. Although the occlusion "cure" rate at median 6-month follow-up is $61 \%$, reflecting an aggregate progressive thrombosis relative to initial angiographic occlusion rate of $45 \%$, the long-term angiographic outcome is somewhat discouraging, particularly considering the relatively high prevalence of "small" aneurysms within our literature pool. Further characterization of occlusion rates with respect to relevant aneurysm complexity parameters could not be accomplished due to incomplete and heterogeneous reporting practices, though there is clear documentation from larger individual series ${ }^{16}$ that increasing aneurysm complexity is associated with significantly higher treatment failure rates. In reviewing the aneurysm demographics, it became evident that stent-assisted coiling, paradoxically, is being applied predominantly for the treatment of small aneurysms that constitute approximately $63 \%$ of the overall cohort. Although this may reflect use of the technique in treating small aneurysms with unfavorable dome-to-neck ratios, it also may suggest expanded use of the technique beyond its initial indication to include aneurysms amenable to direct or balloon-assisted coiling. This possible evolution in the use of stent-assisted coiling is perhaps driven by a perception that stent placement, in some way, improves the long-term angiographic outcome compared with coiling alone. This raises a potentially important, and as yet incompletely answered, question related to the necessity of aneurysm occlusion in realizing the goals of endovascular aneurysm treatment: the prevention of bleeding and rebleeding and relief of symptomatic mass effect. ${ }^{1,48,49}$ In this 
Analysis of stent-related issues in a subgroup of publications containing data on each specific patient

\begin{tabular}{lcrl}
\hline & First 10 & $11+$ & OR \\
\hline No. patients & \multicolumn{1}{c}{100} & \multicolumn{1}{c}{ 240 } & \\
\% total complications & 21.1 & 16.0 & $0.7<\mathrm{OR}<2.5$ \\
\% death & 1.1 & 1.4 & $0.5<\mathrm{OR}<4.2$ \\
\% overall stent problem & 10.0 & 3.1 & $1.3<\mathrm{OR}<6.6^{*}$ \\
\% stent delivery failure & 5.0 & 1.2 & $1.2<\mathrm{OR}<8.7^{*}$ \\
\% stent problem other than delivery & 5.0 & 2.0 & $0.8<\mathrm{OR}<5.3$
\end{tabular}

$\%$ stent problem other than delivery

failure (eg, migration, improper

position)

Note:-The statistics of first 10 patients treated with stent/coils is compared with that of subsequent cases.

* indicates statistically significant.

regard, the prevalence of incomplete occlusion in our cohort is somewhat disappointing, especially considering the additional technical complexity of stent-assisted coiling.

The possibilities of delayed in-stent stenosis and occlusion have assumed greater importance as the adjunctive use of stents has gained wider acceptance in the treatment of relatively "uncomplicated" aneurysms, a trend suggested by the percentage of small-sized aneurysms treated in our study cohort. In the relatively short follow-up period ( 6 months median) we found a rather small incidence of stenosis reported. Nevertheless, $\sim 1 \%$ of stented vessels seem to be occluded at follow-up. Most stenoses and occlusions appear to be clinically silent — in our analysis only 4 of 24 (17\%) stenoses and 1 of 4 occlusions were symptomatic. Although there is some evidence to suggest that both stenosis and occlusion occur relatively early after stent implantation ${ }^{5,9,50}$ and that some untreated stenoses appear to spontaneously resolve on continued follow-up, ${ }^{9,50}$ long-term (3-5-year range) angiographic data are required to better understand the natural history of this process. Clinical follow-up data, showing a 3\% risk of TIA or stroke in the stent-coiled territory, also merit more rigorous long-term study.

\section{Conclusions}

We have compiled the largest to date literature survey of stentsupported aneurysm coiling. Although the reporting of clinical and angiographic follow-up is heterogeneous, the results presented here suggest that stents can be used adjunctively with endosaccular coiling by well-trained operators at the potential price of somewhat increased risks of thromboembolism and parenchymal hemorrhage. The incidence of stentrelated difficulties seems to decrease significantly as operators gain experience with these devices. Ultimately, it seems that approximately $61 \%$ of aneurysms, many displaying unfavorable anatomic characteristics and less amenable to other treatment modalities, can be occluded with stent-assisted coiling. In the future, next-generation endoluminal devices will probably further expand the scope and effectiveness of endovascular aneurysm treatment.

Disclosures: Tibor Becske. Other Financial Relationships: ev3, Details: I have acted as proctor outside the United States for Pipeline cases; I receive a per diem honorarium for this activity and reimbursement of travel-related expenses from ev3. Peter Kim Nelson: Consultant: Chestnut, ev3-Covidien, Details: Consultant and proctor for Pipeline embolization device; Ownership Interest: Chestnut, Surpass, Details: Past shareholder of Chestnut Medical $(>1 \%,<10 \%$ of company's equity); shares sold $6 / 09$; spouse is current shareholder of Surpass Medical ( $>1 \%,<10 \%$ of company's equity).

\section{References}

1. Johnston SC, Dowd CF, Higashida RT, et al. Predictors of rehemorrhage after treatment of ruptured intracranial aneurysms: the Cerebral Aneurysm Rerupture After Treatment (CARAT) study. Stroke 2008;39:120-25

2. Akpek S, Arat A, Morsi H, et al. Self-expandable stent-assisted coiling of widenecked intracranial aneurysms: a single-center experience. AJNR Am J Neuroradiol 2005;26:1223-31

3. Biondi A, Janardhan V, Katz JM, et al. Neuroform stent-assisted coil embolization of wide-neck intracranial aneurysms: strategies in stent deployment and midterm follow-up. Neurosurgery 2007;61:460-68

4. Juszkat R, Nowak S, Smól S, et al. Leo stent for endovascular treatment of broad-necked and fusiform intracranial aneurysms. Interv Neuroradiol 2007;13:255-69

5. Kis B, Weber W, Berlit P, et al. Elective treatment of saccular and broad-necked intracranial aneurysms using a closed-cell nitinol stent (Leo). Neurosurgery 2006;58:443-50

6. Lee YJ, Kim DJ, Suh SH, et al. Stent-assisted coil embolization of intracranial wide-necked aneurysms. Neuroradiology 2005;47:680-89

7. Sedat J, Chau Y, Mondot L, et al. Endovascular occlusion of intracranial widenecked aneurysms with stenting (Neuroform) and coiling: mid-term and long-term results. Neuroradiology 2009;51:401-09

8. Wajnberg E, de Souza JM, Marchiori E, et al. Single-center experience with the Neuroform stent for endovascular treatment of wide-necked intracranial aneurysms. Surg Neurol 2009;72:612-19

9. Weber W, Bendszus M, Kis B, et al. A new self-expanding nitinol stent (Enterprise) for the treatment of wide-necked intracranial aneurysms: initial clinical and angiographic results in 31 aneurysms. Neuroradiology 2007;49:555-61

10. Yahia AM, Gordon V, Whapham J, et al. Complications of Neuroform stent in endovascular treatment of intracranial aneurysms. Neurocrit Care 2008;8:19-30

11. Yavuz K, Geyik S, Saatci I, et al. WingSpan stent system in the endovascular treatment of intracranial aneurysms: clinical experience with midterm follow-up results. J Neurosurg 2008;109:445-53

12. Alfke K, Straube T, Dörner L, et al. Treatment of intracranial broad-neck aneurysms with a new self-expanding stent and coil embolization. AJNR Am J Neuroradiol 2004;25:584-91

13. Benitez RP, Silva MT, Klem J, et al. Endovascular occlusion of wide-necked aneurysms with a new intracranial microstent (Neuroform) and detachable coils. Neurosurgery 2004;54:1359-67; discussion 1368

14. Brisman JL, Song JK, Niimi Y, et al. Treatment options for wide-necked intracranial aneurysms using a self-expandable hydrophilic coil and a self-expandable stent combination. AJNR Am J Neuroradiol 2005;26:1237-40

15. dos Santos Souza MP, Agid R, Willinsky RA, et al. Microstent-assisted coiling for wide-necked intracranial aneurysms. Can J Neurol Sci 2005;32:71-81

16. Fiorella D, Albuquerque FC, Woo H, et al. Neuroform stent-assisted aneurysm treatment: evolving treatment strategies, complications, and results of long term follow-up. JNIS 2010;2:16-22

17. Han PP, Albuquerque FC, Ponce FA, et al. Percutaneous intracranial stent placement for aneurysms. J Neurosurg 2003;99:23-30

18. Hauck EF, Welch BG, White JA, et al. Stent/coil treatment of very large and giant unruptured ophthalmic and cavernous aneurysms. Surg Neurol 2009;71: 19-24; discussion 24

19. Henkes H, Bose A, Felber S, et al. Endovascular coil occlusion of intracranial aneurysms assisted by a novel self-expandable nitinol microstent (Neuroform). Interv Neuroradiol 2002;8:107-19

20. Higashida RT, Halbach VV, Dowd CF, et al. Initial clinical experience with a new self-expanding nitinol stent for the treatment of intracranial cerebral aneurysms: the Cordis Enterprise stent. AJNR Am J Neuroradiol 2005;26:1751-56

21. Horowitz M, Levy E, Sauvageau E, et al. Intra/extra-aneurysmal stent placement for management of complex and wide-necked-bifurcation aneurysms: eight cases using the waffle cone technique. Neurosurgery 2006;58:ONS-258262; discussion ONS-262

22. Jabbour P, Koebbe C, Veznedaroglu E, et al. Stent-assisted coil placement for unruptured cerebral aneurysms. Neurosurg Focus 2004;17:E10

23. Katsaridis V, Papagiannaki C, Violaris C. Embolization of acutely ruptured and unruptured wide-necked cerebral aneurysms using the Neuroform 2 stent without pretreatment with antiplatelets: a single center experience. AJNR Am J Neuroradiol 2006;27:1123-28

24. Lanzino G, Wakhloo AK, Fessler RD, et al. Efficacy and current limitations of intravascular stents for intracranial internal carotid, vertebral, and basilar artery aneurysms. J Neurosurg 1999;91:538-46

25. Liu JM, Huang QH, Xu Y, et al. Combined stent and coil in endovascular treatment of intracranial wide-necked and fusiform aneurysms. Chin Med J (Engl) 2004;117:54-57

26. Lubicz B, Leclerc X, Levivier M, et al. Retractable self-expandable stent for endovascular treatment of wide-necked intracranial aneurysms: preliminary experience. Neurosurgery 2006;58:451-57

27. Lubicz B, François O, Levivier M, et al. Preliminary experience with the Enterprise stent for endovascular treatment of complex intracranial aneurysms: 
potential advantages and limiting characteristics. Neurosurgery 2008;62:106369; discussion 1069-70

28. Luo CB, Wei CJ, Chang FC, et al. Stent-assisted embolization of internal carotid artery aneurysms. J Chin Med Assoc 2003;66:460-66

29. Lylyk P, Ferrario A, Pasbón B, et al. Buenos Aires experience with the Neuroform self-expanding stent for the treatment of intracranial aneurysms. $\mathrm{J} \mathrm{Neu}$ rosurg 2005;102:235-41

30. Maldonado IL, Machi P, Costalat V, et al. Neuroform stent-assisted coiling of unruptured intracranial aneurysms: short- and midterm results from a single-center experience with 68 patients. AJNR Am J Neuroradiol 2011;32:131-36

31. Mocco J, Snyder KV, Albuquerque FC, et al. Treatment of intracranial aneurysms with the Enterprise stent: a multicenter registry. J Neurosurg 2009;110:35-39

32. Piotin M, Blanc R, Spelle L, et al. Stent-assisted coiling of intracranial aneurysms: clinical and angiographic results in 216 consecutive aneurysms. Stroke 2010;41:110-15

33. Sani S, Jobe KW, Lopes DK. Treatment of wide-necked cerebral aneurysms with the Neuroform2 Treo stent. A prospective 6-month study. Neurosurg Focus 2005; $18: \mathrm{E} 4$

34. Siddiqui MA, J Bhattacharya J, Lindsay KW, et al. Horizontal stent-assisted coil embolisation of wide-necked intracranial aneurysms with the Enterprise stent-a case series with early angiographic follow-up. Neuroradiology 2009;51:411-18

35. Szikora I, Berentei Z, Kulcsar Z, et al. Endovascular treatment of intracranial aneurysms with parent vessel reconstruction using balloon and self expandable stents. Acta Neurochir (Wien) 2006;148:711-23; discussion 723

36. Thorell WE, Chow MM, Woo HH, et al. Y-configured dual intracranial stentassisted coil embolization for the treatment of wide-necked basilar tip aneurysms. Neurosurgery 2005;56:1035-40

37. Wanke I, Doerfler A, Goericke S, et al. Treatment of wide-necked intracranial aneurysms with a self-expanding stent: mid-term results. Zentralbl Neurochir 2005;66:163-69

38. Yang $\mathrm{X}, \mathrm{Wu} \mathrm{Z}, \mathrm{Mu} \mathrm{S}$, et al. Endovascular treatment of giant and large intracranial aneurysms using the Neuroform stent-assisted coil placement. Neurol Res 2008;30:598-602

39. Yavuz K, Geyik S, Pamuk AG, et al. Immediate and midterm follow-up results of using an electrodetachable, fully retrievable SOLO stent system in the endovascular coil occlusion of wide-necked cerebral aneurysms. J Neurosurg 2007;107:49-55

40. Zenteno M, Modenesi Freitas JM, Aburto-Murrieta Y, et al. Balloon-expandable stenting with and without coiling for wide-neck and complex aneurysms. Surg Neurol 2006;66:603-10; discussion 610

41. Shapiro M, Babb J, Becske T, et al. Safety and efficacy of adjunctive balloon remodeling during endovascular treatment of intracranial aneurysms: a literature review. AJNR Am J Neuroradiol 2008;29:1777-81

42. Henkes H, Fischer S, Weber W, et al. Endovascular coil occlusion of 1811 intracranial aneurysms: early angiographic and clinical results. Neurosurgery 2004;54:268-80; discussion 280-65

43. Roy D, Milot G, Raymond J. Endovascular treatment of unruptured aneurysms. Stroke 2001;32:1998-2004

44. Park HK, Horowitz M, Jungreis C, et al. Periprocedural morbidity and mortality associated with endovascular treatment of intracranial aneurysms. AJNR Am J Neuroradiol 2005;26:506-14

45. Ross IB, Dhillon GS. Complications of endovascular treatment of cerebral aneurysms. Surg Neurol 2005;64:12-18; discussion 18-19

46. Sluzewski M, Bosch JA, van Rooij WJ, et al. Rupture of intracranial aneurysms during treatment with Guglielmi detachable coils: incidence, outcome, and risk factors. J Neurosurg 2001;94:238-40

47. White PM, Lewis SC, Nahser H, et al. HydroCoil Endovascular Aneurysm Occlusion and Packing Study (HELPS trial): procedural safety and operatorassessed efficacy results. AJNR Am J Neuroradiol 2008;29:217-23

48. Molyneux A, Kerr R, Stratton I, et al. International Subarachnoid Aneurysm Trial (ISAT) of neurosurgical clipping versus endovascular coiling in 2143 patients with ruptured intracranial aneurysms: a randomised trial. Lancet 2002;360:1267-74

49. Molyneux AJ, Kerr RS, Birks J, et al. Risk of recurrent subarachnoid haemorrhage, death, or dependence and standardised mortality ratios after clipping or coiling of an intracranial aneurysm in the International Subarachnoid Aneurysm Trial (ISAT): long-term follow-up. Lancet Neurol 2009;8:427-33

50. Fiorella D, Albuquerque FC, Woo H, et al. Neuroform in-stent stenosis: incidence, natural history, and treatment strategies. Neurosurgery 2006;59: $34-42$ 